\title{
Antenna Coupling Error in Direction Finders ${ }^{1}$
}

\author{
Charles W. Harrison, Jr.
}

\author{
(August 15, 1960; revised January 19, 1961)
}

\begin{abstract}
Antenna coupling or scattering error in an interferometer-angle-measuring system consisting of two identical base-loaded quarter-wave vertical antennas over a perfectly conducting earth is investigated. A curve is supplied for the error in angle as a function of antenna spacing for an incident vertically polarized electric field arriving in the plane of the antennas, when the ratio of antenna length to radius is 75 and the base loads are resistors of $50 \mathrm{ohms}$.
\end{abstract}

\section{Introduction}

An interferometer-angle-measuring system for missile trajectory determination - in rudimentary form - consists of four identical base-loaded vertical antennas, one-quarter wave or less in length, erected at the vertices of a square. The phase angles between the voltages developed across identical load impedances in diagonal antenna pairs are measured. These angles establish the direction cosines of the missile from which the signal emanates. In addition to electronic missile tracking equipment, many fixed antenna direction finders utilize this principle.

There are several sources of error in interferometerangle-measuring systems. It is convenient to class these errors under two headings: geometrical and electrical. Simmons [1959] has studied geometrical errors in a radio interferometer. Under electrical errors, Carswell and Flammer [1957] investigated the error due to multipath. In the present paper antenna coupling error in a two-antenna interferometer is investigated theoretically and numerically. The model employed consists of two isolated parallel nonstaggered antennas of the same length and radius, center-loaded by identical impedances. The incident electric field is assumed to be polarized parallel to the axes of the antennas, but this restriction in the theory may be removed. The azimuth angle of wave arrival that is indicated by the equipment is computed and compared to the assumed angle of wave arrival. The computed error angle then applies to a base-loaded two-antenna array over a perfectly conducting earth for which the loading impedances are halved. Second order accuracy is achieved in the theoretical development and in the numerical results of this report. In another paper Harrison [1960] studied the more general case of the four-antenna direction finder and obtained results accurate to zeroth order.

Some insight into the nature of antenna coupling error may be gained from the following remarks: Each antenna in the array continuously samples the scattered field from the other antennas comprising the array. The resultant field acting on each antenna in the interferometer is the vector sum of the feeld maintained along its surface by the distant telemetry transmitter on the missile and the several scattered

\footnotetext{
1 Contribution from the Sandia Laboratory, Albuqerque, N. Mex.
}

fields maintained by the currents in the other antennas. Thus the coordinates of the missile, determined by the phase-comparison method, must be in error. The error is a function of the polarization of the electric field, the coordinates of the signal source, the antenna dimensions (length and radius), the spacing between elements, the load impedance, and the properties of the earth. In this paper the scattering error in an interferometer that is far from the earth, or that is erected on a very highly conducting plane, is determined. The effect of an imperfectly conducting earth is not treated.

\section{Integral Equations for the Currents in an Unloaded Two-Element Receiving Array and Their Solutions}

Consider two identical non-staggered parallel receiving antennas, labeled 1 and 2 , oriented as shown in figure 1 with respect to a system of rectangular

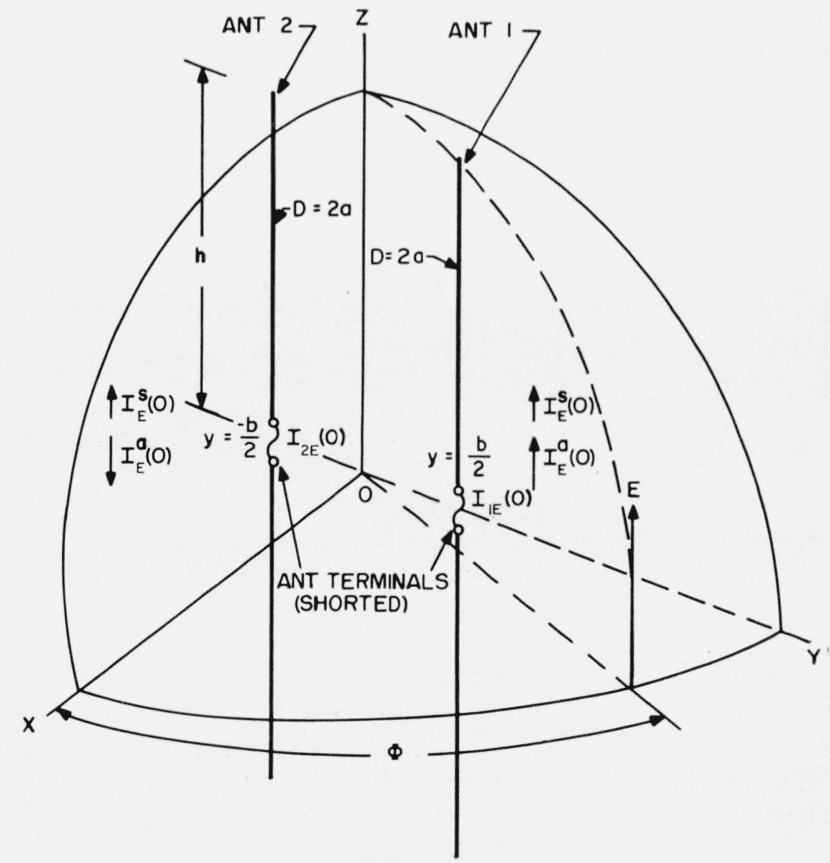

FiguRE 1. Two-element receiving antenna array (load terminals short-circuited). 
and spherical coordinates with common origin. The half-length of each antenna is $h$, the radius $a$, and the spacing between them is $b$. It is assumed that $h>>a$, and $\beta a<<1$. Here $\beta$ is the radian wave number. The terminals of the receiving elements are located at $x=0, y= \pm \frac{b}{2}$, and $z=0$. The incident electric field $E$ arrives from the distant source at the azimuth angle $\Phi$, measured from the positive $x$-axis, and is directed parallel to the axes of the wires. The currents in antennas 1 and 2 , when the terminals are short-circuited, are designated $I_{1 E}(z)$ and $I_{2 E}(z)$, respectively, and are functions of $E, \Phi$, and the electrical dimensions of the array $\beta a, \beta h$, and $\beta b$. From symmetry considerations it is clear that $I_{1 E}(z) \neq I_{2 E}(z)$ except when $\Phi=n \pi, n=0,1$, 2 ....

When the electric field is directed parallel to the axes of the wires, the simultaneous integral equations for determining the currents $I_{1 E}(z)$ and $I_{2 E}(z)$ are [King, 1956, p. 517, eqs (2a) and (2b)]

$$
\begin{array}{r}
\int_{-h}^{h} I_{1 E}\left(z^{\prime}\right) K_{a}\left(z, z^{\prime}\right) d z^{\prime}+\int_{-h}^{h} I_{2 E}\left(z^{\prime}\right) K_{b}\left(z, z^{\prime}\right) d z^{\prime} \\
=-j \frac{4 \pi}{\zeta}\left\{C_{1} \cos \beta z+U e^{j \frac{\beta b}{2} \sin \Phi}\right\}
\end{array}
$$

$$
\begin{array}{r}
\int_{-h}^{h} I_{2 E}\left(z^{\prime}\right) K_{a}\left(z, z^{\prime}\right) d z^{\prime}+\int_{-h}^{h} I_{1 E}\left(z^{\prime}\right) K_{b}\left(z, z^{\prime}\right) d z^{\prime} \\
=-j \frac{4 \pi}{\zeta}\left\{C_{2} \cos \beta z+U e^{-j \frac{\beta b}{2} \sin \Phi}\right\} .
\end{array}
$$

Here

$$
K_{a}\left(z, z^{\prime}\right)=\frac{e^{-j \beta \sqrt{\left(z-z^{\prime}\right)^{2}+a^{2}}}}{\sqrt{\left(z-z^{\prime}\right)^{2}+a^{2}}}
$$

$K_{b}\left(z, z^{\prime}\right)$ is obtained from (3) by writing $b$ for $a$, $\zeta$ is the characteristic resistance of space, $C_{1}$ and $C_{2}$ are constants, and

$$
U=-E / \beta .
$$

The currents $I_{1 E}(z)$ and $I_{2 E}(z)$ may be written

$$
\begin{aligned}
& I_{1 E}(z)=I_{E}^{s}(z)+I_{E}^{a}(z) \\
& I_{2 E}(z)=I_{E}^{s}(z)-I_{E}^{a}(z)
\end{aligned}
$$

where $I_{E}^{s}(z)$ and $I_{E}^{a}(z)$ are the symmetrical and antisymmetrical components of current in either antenna. The assumed directions of these currents are indicated in figure 1.

Substituting (5) and (6) into (1) and (2), the following independent integral equations for the currents $I_{E}^{s}(z)$ and $I_{E}^{a}(z)$ are obtained:

$$
\int_{-h}^{h} I_{E}^{s}\left(z^{\prime}\right) K^{s}\left(z, z^{\prime}\right) d z^{\prime}=-j \frac{4 \pi}{\zeta}\left\{C^{s} \cos \beta z+U^{s}\right\}
$$

$\int_{-h}^{h} I_{E}^{a}\left(z^{\prime}\right) K^{a}\left(z, z^{\prime}\right) d z^{\prime}=-j \frac{4 \pi}{\zeta}\left\{C^{a} \cos \beta z+U^{a}\right\}$.

In (7) and (8),

$$
\begin{gathered}
K^{s}\left(z, z^{\prime}\right)=K_{a}\left(z, z^{\prime}\right)+K_{b}\left(z, z^{\prime}\right), \\
K^{a}\left(z, z^{\prime}\right)=K_{a}\left(z, z^{\prime}\right)-K_{b}\left(z, z^{\prime}\right), \\
U^{s}=U \cos \left(\frac{\beta b}{2} \sin \Phi\right), \\
U^{a}=j U \sin \left(\frac{\beta b}{2} \sin \Phi\right), \\
C^{s}=\frac{C_{1}+C_{2}}{2}, \\
C^{a}=\frac{C_{1}-C_{2}}{2} .
\end{gathered}
$$

The integral equation for the unloaded current in an isolated receiving antenna consisting of a single conductor is identical in form to (7) and (8). It follows that solutions for the currents $I_{E}^{s}(o)$ and $I_{E}^{a}(o)$ may be obtained from investigations of the receiving dipole by appropriate substitutions for the kernel and the $U$-function. These currents may also be obtained from the relations

$$
I_{E}^{s}(0)=-\frac{2 \beta h_{e}^{s} U^{s}}{Z^{s}}=-\frac{2 \beta h_{e}^{s} U \cos \left(\frac{\beta b}{2} \sin \Phi\right)}{Z_{s 1}+Z_{12}},
$$

and

$$
I_{E}^{a}(0)=-\frac{2 \beta h_{e}^{a} U^{a}}{Z^{a}}=-j \frac{2 \beta h_{e}^{a} U \sin \left(\frac{\beta b}{2} \sin \Phi\right)}{Z_{s 1}-Z_{12}}
$$

where $2 h_{e}^{s}$ is the symmetrical effective length of either antenna; $2 h_{e}^{a}$ is the antisymmetrical effective length of either antenna; $Z_{s 1}$ is the self-impedance of either antenna in the presence of the other, and $Z_{12}$ is the mutual impedance. Equations (15) and (16) follow directly from the equivalent circuit of a receiving antenna, for the unloaded condition.

The no-load currents $I_{1 E}(0)$ and $I_{2 E}(0)$ may be obtained from (5) and (6) using (15) and (16). These are

$I_{1 E}(0)=-4 \pi U\left\{\frac{h_{e}^{s}}{\lambda}\left[\frac{\cos \left(\frac{\beta b}{2} \sin \Phi\right)}{Z_{s 1}+Z_{12}}\right]\right.$
$\left.+j \frac{h_{e}^{a}}{\lambda}\left[\frac{\sin \left(\frac{\beta b}{2} \sin \Phi\right)}{Z_{s 1}-Z_{12}}\right]\right\}$, 
and

$$
\begin{aligned}
I_{2 E}(0)=-4 \pi U\left\{\frac{h_{e}^{s}}{\lambda}\right. & {\left[\frac{\cos \left(\frac{\beta j}{2} \sin \Phi\right)}{Z_{s 1}+Z_{12}}\right] } \\
& \left.-j \frac{h_{e}^{a}}{\lambda}\left[\frac{\sin \left(\frac{\beta b}{2} \sin \Phi\right)}{Z_{s 1}-Z_{12}}\right]\right\} .
\end{aligned}
$$

Second order mutual- and self-impedances are available in the literature [King, 1956, ch 3]. If the symmetrical and antisymmetrical effective lengths can be found to the same accuracy, second order no-load currents can be obtained, using (17) and (18). It turns out to be much simpler, from the computational point of view, to determine the symmetrical and antisymmetrical effective lengths to second order accuracy than to evaluate numerically the solutions of the integral eqs (7) and (8) to obtain second order short-circuit currents. A second order solution for the scattering error in direction finders is sought in this paper.

The effective lengths $2 h_{e}^{s}$ and $2 h_{e}^{a}$ are like $2 h_{e}$ for an isolated dipole except that the expansion parameters are $\psi^{s}$ and $\psi^{a}$ instead of $\psi_{K 1}$. These differences should be expected from an examination of the integral eqs (7) and (8). The various expansion parameters are defined as follows when $\beta h=\pi / 2$ :

$$
\psi^{s}=\left|C_{a}\left(\frac{\lambda}{4}, 0\right)+C_{b}\left(\frac{\lambda}{4}, 0\right)\right|
$$

[King, 1956, p. 268, eqs (12) and (14a)],

$$
\psi^{a}=\left|C_{a}\left(\frac{\lambda}{4}, 0\right)-C_{b}\left(\frac{\lambda}{4}, 0\right)\right|
$$

[King, 1956, p. 273, eq (4a)],

$$
\psi_{K 1}=\left|C_{a}\left(\frac{\lambda}{4}, 0\right)\right|
$$

[King, 1956, p. 102, eqs (27) and (29a)].

Here

$$
\begin{gathered}
C_{a}\left(\frac{\lambda}{4}, 0\right) \approx 2 \sinh ^{-1}\left(\frac{h}{a}\right)-\overline{\mathrm{Ci}} \pi-j \operatorname{Si} \pi, \\
C_{b}\left(\frac{\lambda}{4}, 0\right)=\operatorname{Ci} \beta p-\operatorname{Ci} \beta q-j \operatorname{Si} \beta p+j \operatorname{Si} \beta q, \\
p=\sqrt{\left(\frac{\lambda}{4}\right)^{2}+b^{2}}+\frac{\lambda}{4}, \\
q=\sqrt{\left(\frac{\lambda}{4}\right)^{2}+b^{2}}-\frac{\lambda}{4},
\end{gathered}
$$

$\overline{\mathrm{Ci}} X=0.5772+\ln X-\mathrm{Ci} X$.
An important relation is

where

$$
\psi_{K 1}=\Omega-k
$$

$$
\Omega=2 \ln \left(\frac{2 h}{a}\right),
$$

and $k=1.445$ when $7 \leq \Omega \leq 20$ for $\beta h=\frac{\pi}{2}$ [King, 1956, p. 105, fig. 20.7]. It is reasonable to suppose that

and

$$
\psi^{s}=\Omega^{s}-k,
$$

$$
\psi^{a}=\Omega^{a}-k .
$$

[King, 1956, p. 270, fig. 3.3].

From (27) and (29),

$$
\Omega^{s}=\Omega+\psi^{s}-\psi_{K 1},
$$

and from (27) and (30),

$$
\Omega^{a}=\Omega+\psi^{a}-\psi_{K 1} .
$$

$\Omega^{s}$ and $\Omega^{a}$ may now be computed from (31) and (32) using (19), (20), (21), and (28) when $\beta h=\pi / 2$. The values of $\left|h_{e}^{s}\right| / \lambda$ and $\left|h_{e}^{a}\right| / \lambda$ are then read directly from figure 2 , a curve relating $\left|h_{e}\right| / \lambda$ to $\Omega$ when $\beta h=\pi / 2$. It should be mentioned that in general

$$
h_{e}=h_{e}^{\prime \prime}+j h_{e}^{\prime} .
$$

Reference to the literature [King, 1956, p. 488, fig. 9.2a] shows that $h_{e}^{\prime} \approx 0$ for all values of $\Omega$. when $\beta h=\pi / 2$. It is a legitimate procedure, therefore, to replace $h_{e}^{s}$ and $h_{e}^{a}$ by $\left|h_{e}^{s}\right|$ and $\left|h_{e}^{a}\right|$, respectively, in (17) and (18) provided the theory is restricted to the case $\beta h=\pi / 2$.

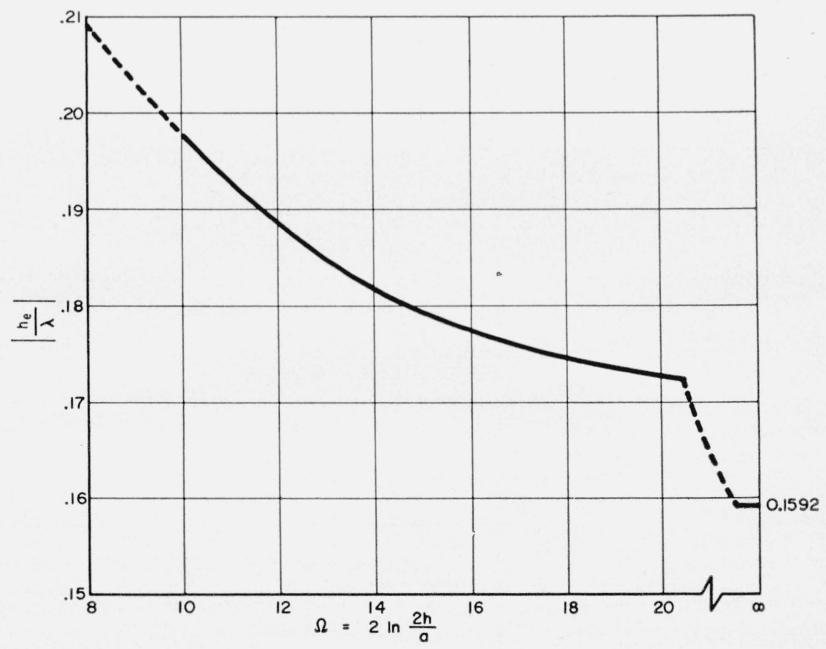

Figure 2. Effective length $\left|\frac{\mathrm{h}_{\mathrm{e}}}{\lambda}\right|$ for a half-wave dipole as a function of $\Omega(\beta \mathrm{h}=\pi / 2)$ 
In table 1 are listed second order values of $Z_{12}$ and $Z_{s 1}$ as functions of $b / \lambda$ when $\beta h=\pi / 2$ and $\Omega=10$ [King, 1956 , p. 305, tables 8.10 and 8.11]. Table 2 provides computed values of $Y^{s}=1 / Z^{s}$ and $Y^{a}=1 / Z^{a}$ for $\beta h=\pi / 2$ and $\Omega=10$. This table facilitates error computations when $\Phi \neq \pi / 2$. Table 3 furnishes computed values of $\psi^{s}, \psi^{a}, \Omega^{s}$, and $\Omega^{a}$ and the values of $\left|h_{e}^{s}\right| / \lambda$ and $\left|h_{e}^{a}\right| / \lambda$, as read from figure 2 when $\Omega=10$ and $\beta h=\pi / 2$. These data permit evaluation of $I_{1 E}(o)$ and $I_{2 E}(o)$ from (17) and (18), as functions of $\Phi$, to second order accuracy.

TABLE 1. Mutual and self-impedance for coupled antennas

$$
\Omega=2 \ln \left(\frac{2 h}{a}\right)=10, \beta h=\frac{\pi}{2}
$$

(All values are in ohms)

\begin{tabular}{r|r|c}
\hline \hline$\frac{b}{\lambda}$ & \multicolumn{1}{|c|}{$Z_{12}$} & $Z_{s 1}$ \\
\cline { 1 - 2 } 0.1 & $75.1-j 7.8$ & \\
\cline { 2 - 3 } .2 & $55.2-j 33.7$ & $82.0+j 37.7$ \\
.3 & $29.9-j 46.3$ & $82.4+j 38.7$ \\
.4 & $4.9-j 43.2$ & $84.6+j 39.9$ \\
.5 & $-15.2-j 30.4$ & $85.8+j 41.7$ \\
.6 & $-26.2-j 12.5$ & $86.2+j 42.9$ \\
.7 & $-27.0+j 4.9$ & $86.1+j 42.9$ \\
.8 & $-19.5+j 17.1$ & $86.2+j 42.8$ \\
.9 & $-7.2+j 21.6$ & $86.3+j 42.8$ \\
1.0 & $5.1+j 18.2$ & $86.4+j 42.9$ \\
\hline
\end{tabular}

TABLe 2. Symmetrical and antisymmetrical admittances for coupled antennas

$$
\beta h=\frac{\pi}{2}, \Omega=10
$$

\begin{tabular}{|c|c|c|}
\hline$\frac{b}{\lambda}$ & $Y^{s}=\frac{1^{*}}{Z^{s}}$ & $Y^{a}=\frac{1^{*}}{Z^{a}}$ \\
\hline $\begin{array}{r}0.1 \\
.2 \\
.3 \\
.4 \\
.5\end{array}$ & $\begin{array}{r}0.6143-\mathrm{j} 0.1169 \\
.7311-\mathrm{j} 0.0268 \\
.8909+\mathrm{j} 0.0510 \\
1.112+\mathrm{j} 0.0187 \\
1.375-\mathrm{j} 0.2395\end{array}$ & 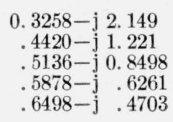 \\
\hline $\begin{array}{r}.6 \\
.7 \\
.8 \\
.9 \\
1.0\end{array}$ & $\begin{array}{r}1.326-\mathrm{j} 0.6720 \\
1.023-\mathrm{j} 0.8273 \\
0.8299-\mathrm{j} 0.7453 \\
.7603-\mathrm{j} 0.6190 \\
.7559-\mathrm{j} 0.5048\end{array}$ & $\begin{array}{rr}.7160-\mathrm{j} & .3529 \\
.7946-\mathrm{j} & .2670 \\
.8933-\mathrm{j} & .2172 \\
1.017-\mathrm{j} & .2307 \\
1.126-\mathrm{j} & .3421\end{array}$ \\
\hline
\end{tabular}

${ }^{*}$ Multiply all numbers by $10^{-2}$

TABIE 3. Computed values of the expansion parameters and the symmetrical and antisymmetrical effective lengths for

\begin{tabular}{|c|c|c|c|c|c|c|}
\hline$\frac{b}{\lambda}$ & $\psi^{8}$ & $\psi^{a}$ & $\Omega^{s}$ & $\Omega a$ & $\left|h_{e}^{s}\right| / \lambda$ & $\left|h_{e}^{a}\right| / \lambda$ \\
\hline $\begin{array}{r}0.1 \\
.2 \\
.3 \\
.4 \\
.5\end{array}$ & $\begin{array}{r}10.55 \\
9.18 \\
8.41 \\
8.03 \\
7.94\end{array}$ & $\begin{array}{l}6.78 \\
8.12 \\
8.81 \\
9.12 \\
9.17\end{array}$ & $\begin{array}{r}12.00 \\
10.63 \\
9.86 \\
9.48 \\
9.39\end{array}$ & $\begin{array}{r}8.23 \\
9.57 \\
10.26 \\
10.57 \\
10.62\end{array}$ & $\begin{array}{r}0.1884 \\
.1944 \\
.1984 \\
.2005 \\
.2019\end{array}$ & $\begin{array}{r}0.2077 \\
.2000 \\
.1964 \\
.1948 \\
.1944\end{array}$ \\
\hline $\begin{array}{r}.6 \\
.7 \\
.8 \\
.9 \\
1.0\end{array}$ & $\begin{array}{l}8.09 \\
8.36 \\
8.62 \\
8.81 \\
8.87\end{array}$ & $\begin{array}{l}9.02 \\
8.77 \\
8.52 \\
8.32 \\
8.25\end{array}$ & $\begin{array}{r}9.54 \\
9.81 \\
10.10 \\
10.25 \\
10.32\end{array}$ & $\begin{array}{r}10.47 \\
10.22 \\
9.97 \\
9.77 \\
9.70\end{array}$ & $\begin{array}{l}.2002 \\
.1988 \\
.1975 \\
.1964 \\
.1961\end{array}$ & $\begin{array}{l}.1955 \\
.1965 \\
.1979 \\
.1990 \\
.1995\end{array}$ \\
\hline
\end{tabular}
$\beta \mathrm{h}=\frac{\pi}{2}$ and $\Omega=10$

\section{Circuital Relations in Coupled Receiving Antennas}

Given the short-circuit currents $I_{1 E}(o)$ and $I_{2 E}(o)$, the problem is that of finding the voltage $V_{1 L}$ and $V_{2 L}$ that is developed across identical load impedances $Z_{L}$ connected across the terminals in antennas 1 and 2, respectively. Writing $V_{1 L}=\left|V_{1 L}\right| / \phi_{1 L}$ and $\mathrm{V}_{2 L}=\left|V_{2 L}\right| / \phi_{2 L}$, the angle sought is $\phi=\phi_{1 L}-\phi_{2 L}$. The angular error in the interferometer due to antenna coupling or scattering is then

$$
\delta=\phi-\beta b \sin \Phi .
$$

The terminal currents in a two-element transmitting array immersed in an electric field may be obtained by superposition, as indicated in figure 3. Clearly

and

$$
I_{1 L}(0)=I_{1 E}(0)+I_{1 V}(0)
$$

$$
I_{2 L}(0)=I_{2 E}(0)+I_{2 V}(0)
$$

Here $I_{1 E}(0)$ and $I_{2 E}(0)$ are the terminal currents when the antennas are excited only by the incident electric field, and the impedanceless generator voltages $V_{1}$ and $V_{2}$ are zero; $I_{1 V}(0)$ and $I_{2 V}(0)$ are the terminal currents when the incident electric fields $E_{1}$ and $E_{2}$ are zero and the antennas are center-driven by the voltages $V_{1}$ and $V_{2}$.

The fields, currents and voltages shown in figure 3 are separated into symmetrical and antisymmetrical parts as follows:

$$
\begin{aligned}
E_{1} & =E^{s}+E^{a} \\
E_{2} & =E^{s}-E^{a} \\
I_{1 L}(0) & =I_{L}^{s}(0)+I_{L}^{a}(0) \\
I_{2 L}(0) & =I_{L}^{s}(0)-I_{L}^{a}(0) \\
V_{1} & =V^{s}+V^{a} \\
V_{2} & =V^{s}-V^{a}
\end{aligned}
$$

This separation permits the symmetrical problem (superscript $s$ ) portrayed by figure 4 to be discussed independently of the antisymmetrical problem (superscript $a$ ) portrayed by figure 5 .

\section{Symmetrical Problem}

Refer to figure 4. The following relations are true:

$$
\begin{gathered}
I_{L}^{s}(0)=I_{E}^{s}(0)+I_{V}^{s}(0), \\
I_{V}^{s}(0)=\frac{V^{s}}{Z^{s}}, \\
V^{s}=-I_{L}^{s}(0) Z_{L}, \\
Z^{s}=Z_{s 1}+Z_{12} .
\end{gathered}
$$




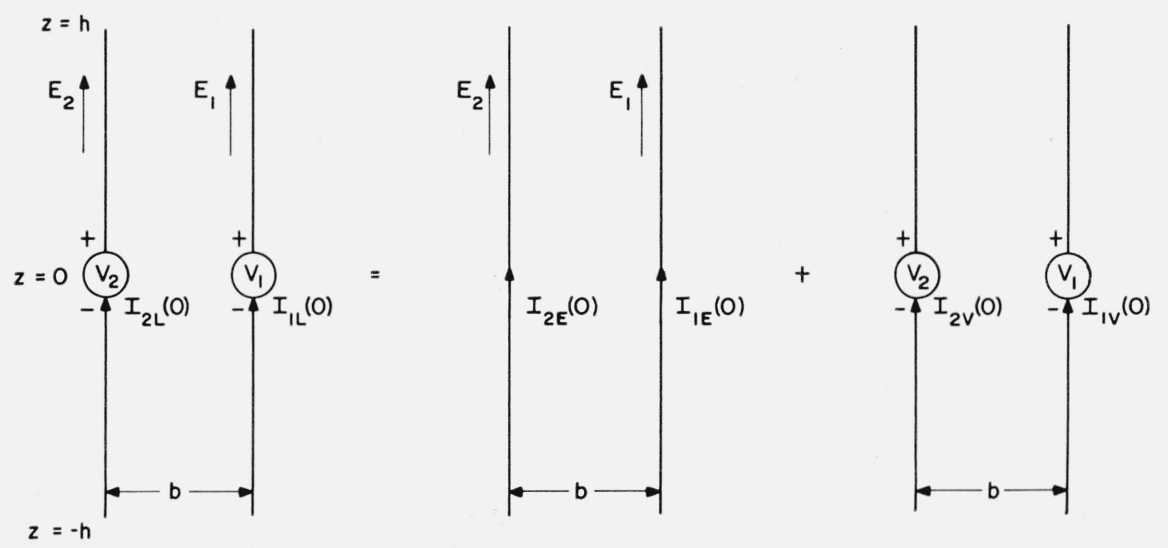

FIGURE 3. The principle of superposition applied to obtain the terminal currents in a two-element transmitting array immersed in an electric field.

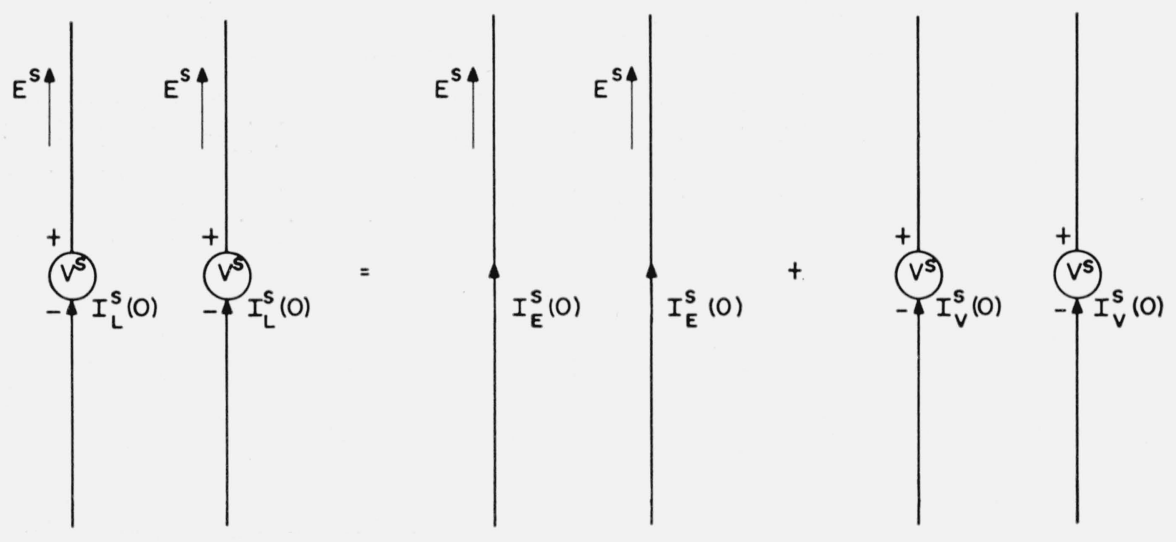

FIgURE 4. The symmetrical problem.

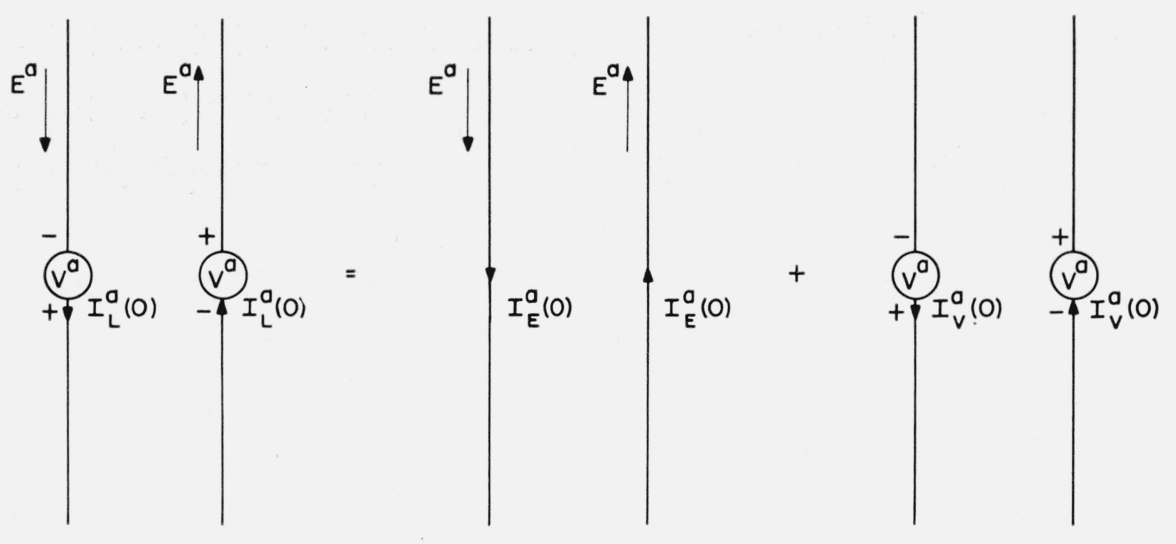

Figure 5. The antisymmetrical problem.

367 
Hence

$$
I_{L}^{s}(0)=I_{E}^{s}(0)\left\{\frac{Z_{s 1}+Z_{12}}{Z_{s 1}+Z_{12}+Z_{L}}\right\}
$$

Equation (47) establishes the relation between the symmetrical part of the load current and the symmetrical part of the short-circuit current.

\section{Antisymmetrical Problem}

Refer to figure 5. The relations (43) to (45) apply with superscript $a$ written for $s$. The antisymmetrical impedance is required. It is

It follows that

$$
Z^{a}=Z_{s 1}-Z_{12}
$$

$$
I_{L}^{a}(0)=I_{E}^{a}(0)\left\{\frac{Z_{s 1}-Z_{12}}{Z_{s 1}-Z_{12}+Z_{L}}\right\}
$$

Equation (49) establishes the relation between the antisymmetrical part of the load current, and the antisymmetrical part of the short-circuit current.

Figure 6 illustrates the two-element receiving antenna array with identical impedances $Z_{L}$ connected to the load terminals of each antenna. The load currents in antennas 1 and 2 are given by (39) and (40), respectively. The voltage drop across $Z_{L}$ in antenna 1 is then

$$
V_{1 L}=Z_{L}\left\{I_{L}^{s}(0)+I_{L}^{a}(0)\right\},
$$

and the voltage drop across $Z_{L}$ in antenna 2 is

$$
V_{2 L}=Z_{L}\left\{I_{L}^{s}(0)-I_{L}^{a}(0)\right\} .
$$

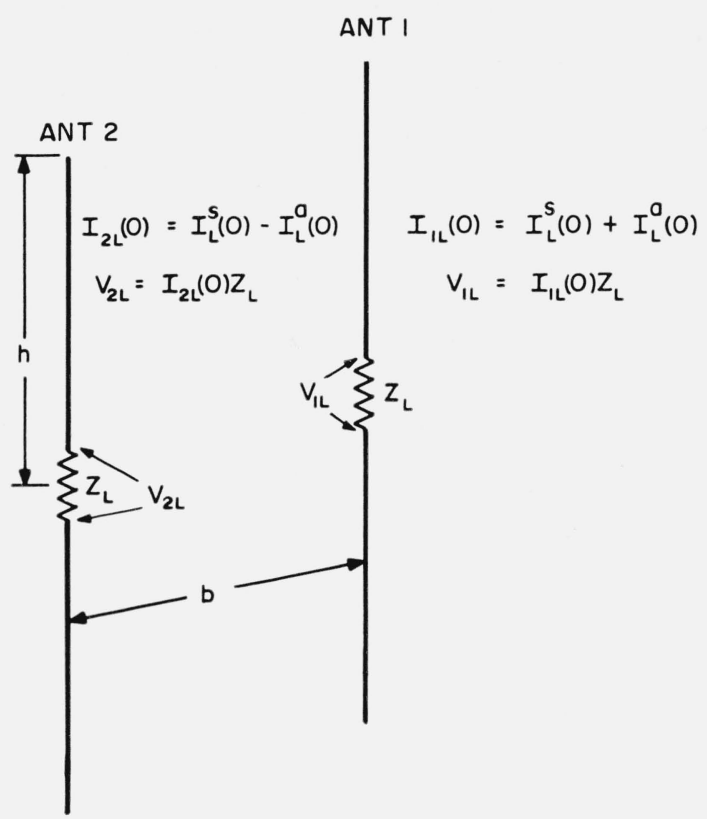

Figure 6. Two-element receiving antenna array. Impedances of value $Z_{L}$ are connected to the load terminals.
$I_{L}^{s}(0)$ and $I_{L}^{a}(0)$ are expressed in terms of $I_{E}^{s}(0)$ and $I_{E}^{a}(0)$ by (47) and (49). Also, from (5) and (6), $\quad I_{E}^{s}(0)=\left(I_{1 E}(0)+I_{2 E}(0)\right) / 2$ and $I_{E}^{a}(0)=\left(I_{1 E}(0)\right.$ $\left.-I_{2 E}(0)\right) / 2$. It is now a simple matter to obtain $V_{1 L}$ and $V_{2 L}$, as given by (50) and (51), in terms of $I_{1 E}(0)$ and $I_{2 E}(0)$. The final expressions are

$$
\begin{aligned}
V_{1 L}= & \frac{Z_{L}}{\left(Z_{s 1}+Z_{12}+Z_{L}\right)\left(Z_{s 1}-Z_{12}+Z_{L}\right)} \\
& \left\{I_{1 E}(0)\left[Z_{s 1}^{2}+Z_{s 1} Z_{L}-Z_{12}^{2}\right]+I_{2 E}(0) Z_{12} Z_{L}\right\}
\end{aligned}
$$

and

$$
\begin{aligned}
V_{2 L}= & \frac{Z_{L}}{\left(Z_{s 1}+Z_{12}+Z_{L}\right)\left(Z_{s 1}-Z_{12}+Z_{L}\right)} \\
& \left\{I_{2 E}(0)\left[Z_{s 1}^{2}+Z_{s 1} Z_{L}-Z_{12}^{2}\right]+I_{1 E}(0) Z_{12} Z_{L}\right\}
\end{aligned}
$$

These equations provide a way of computing the voltages across the identical load impedances in terms of the short-circuit currents $I_{1 E}(0)$ and $I_{2 E}(0)$.

One check on the validity of either of the above expressions is to let $b \rightarrow \infty$ so that $Z_{12} \rightarrow 0$. Then (52), for example, becomes

$$
V_{1 L}^{\prime}=\frac{I_{1 E}^{\prime}(0) Z_{s 1}^{\prime} Z_{L}}{Z_{s 1}^{\prime}+Z_{L}}
$$

Here the primes indicate that the various parameters change value when one of the antennas (in this case antenna 2) is moved to infinity. Equation (54) is recognized immediately as the correct expression for the voltage across the load impedance $Z_{L}$ of an isolated center-loaded receiving antenna.

Let the following definitions be made:

$$
\left.\begin{array}{l}
\frac{Z_{L}}{\left(Z_{s 1}+Z_{12}+Z_{L}\right)\left(Z_{s 1}-Z_{12}+Z_{L}\right)}=\Gamma \\
Z_{s 1}^{2}+Z_{s 1} Z_{L}-Z_{12}^{2}=A e^{j \alpha} \\
Z_{12} Z_{L}=B e^{j \beta} \\
I_{2 E}(0)=C I_{1 E}(0) e^{j \gamma}
\end{array}\right\} .
$$

With this notation (52) and (53) become

$$
\begin{gathered}
V_{1 L}=\Gamma I_{1}(0)\left\{A e^{j \alpha}+B C e^{j(\beta+\gamma)}\right\} \\
V_{2 L}=\Gamma I_{1}(0)\left\{A C e^{j(\alpha+\gamma)}+B e^{j \beta}\right\} .
\end{gathered}
$$

Using these expressions and the formula

$$
\tan ^{-1} x-\tan ^{-1} y=\tan ^{-1}\left\{\frac{x-y}{1+x y}\right\}
$$

it is readily deduced that the angle $\phi$ indicated by the interferometer is

$$
\phi=\tan ^{-1}\left\{\frac{\left(B^{2}-A^{2}\right) C \sin \gamma+A B\left(1-C^{2}\right) \sin (\alpha-\beta)}{\left(B^{2}+A^{2}\right) C \cos \gamma+A B\left(1+C^{2}\right) \cos (\alpha-\beta)}\right\}
$$




\section{Numerical Analysis}

Figure 7 exhibits a second order curve for $\delta$ as a function of $\beta b$ for an isolated interferometer when $\Omega=10, \beta h=\pi / 2, \Phi=\pi / 2$ and $Z_{L}=100+j 0.0$ ohms. To facilitate computations for other values of $\Phi$ and $Z_{L}$, when $\Omega=10$ and $\beta h=\pi / 2$, tables 2 and 3 are provided. Table 4 , giving values of $A, \alpha, B$ and $\beta$ as a function of $b / \lambda$ is useful when constructing curves of $\delta$ against $\beta b$ with $\Phi$ as parameter. This table applies only when $\Omega=10, \beta h=\pi / 2$ and $Z_{L}=100+j 0.0 \mathrm{ohms}$.

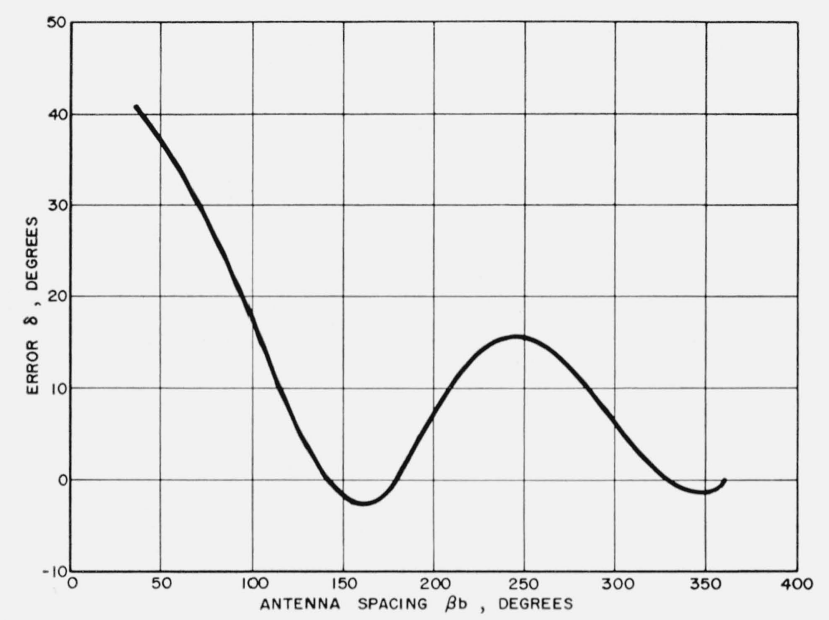

FiguRE 7. Antenna coupling error in a two-antenna interferometer.

$\Omega=10, \boldsymbol{\beta} h=\pi / 2, \Phi=\pi / 2$, and $Z_{L}=100+\mathrm{j} 0.0 \mathrm{ohms}$.

TABLE 4. A, $\alpha, \mathrm{B}$ and $\beta$ versus $\frac{\mathrm{b}}{\lambda}$

(These data apply only when $\beta h=\pi / 2, \Omega=2 \ln (2 h / a)=10$ and $Z_{L}=100+j 0.0 \mathrm{ohms}$ )

\begin{tabular}{|c|c|c|c|c|c|}
\hline$\frac{b}{\lambda}$ & $\boldsymbol{\beta} b$ & $A^{*}$ & $\alpha$ & $B^{*}$ & $\beta$ \\
\hline $\begin{array}{r}0.1 \\
.2 \\
.3 \\
.4 \\
.5\end{array}$ & $\begin{array}{l}0.6283 \\
1.257 \\
1.885 \\
2.513 \\
3.142\end{array}$ & $\begin{array}{l}1.366 \\
\text { 1. } 794 \\
1.974 \\
1.956 \\
1.826\end{array}$ & $\begin{array}{r}0.9520 \\
.8853 \\
.7396 \\
.6371 \\
.6245\end{array}$ & $\begin{array}{r}0.7551 \\
.6467 \\
.5512 \\
.4348 \\
.3399\end{array}$ & $\begin{array}{c}-0.1037 \\
-.5479 \\
-.9975 \\
-1.458 \\
-2.034\end{array}$ \\
\hline $\begin{array}{r}.6 \\
.7 \\
.8 \\
.9 \\
1.0\end{array}$ & $\begin{array}{l}3.770 \\
4.398 \\
5.027 \\
5.655 \\
6.283\end{array}$ & $\begin{array}{l}1.757 \\
1.801 \\
1.875 \\
1.893 \\
1.857\end{array}$ & $\begin{array}{r}.6783 \\
.7252 \\
.7174 \\
.6849 \\
.6687\end{array}$ & $\begin{array}{r}.2903 \\
.2744 \\
.2593 \\
.2277 \\
.1890\end{array}$ & $\begin{array}{r}-2.696 \\
2.962 \\
\text { 2. } 422 \\
1.893 \\
1.300\end{array}$ \\
\hline
\end{tabular}

${ }^{*}$ Multiply $A$ and $B$ values by $10^{4}$.

\section{Concluding Remarks}

It is of interest to observe that when $\Phi=\pi / 2, \delta=0$ for $\beta b=n \pi, n=0,1,2, \ldots$ Evidently, when $\Phi \neq \pi / 2$ a spacing $\beta b$ exists such that $\delta=0$.

The error curve shown in figure 7 applies to baseloaded vertical antennas over a perfectly conducting earth when the load resistors are halved. If all impedances are divided by $2, \alpha, \beta, \gamma$, and $C$ remain unchanged, but $A$ and $B$ are one-fourth their free space values. Accordingly $\phi$ (and therefore $\delta$ ) are the same as for the isolated array. These statements are readily verified using (55) and (59).

A precise theory has been presented for the scattering error in a two-antenna direction finder. It applies with equal accuracy to radio interferometers employing antennas of length other than $h=\lambda / 4$ (but with slight increase in complexity). Furthermore, the theory is readily generalized to include a wave tilt with respect to the antenna axes.

The writer is indebted to R. W. P. King of Harvard University, and R. H. Duncan of New Mexico State University, for reading this paper. He wishes to thank Mrs. Dixie S. Baca for doing the typing.

\section{References}

Carswell, I., and C. Flammer, Ground antenna phase behavior in a differential phase measuring system, Part I 1957 IRE National Convention Record, pp. 49-56.

Harrison, C. W., Jr., Scattering error in electronic missile trajectory measuring equipment, SCDC-1084 of Sept. 1, 1960 (unpublished).

King, Ronold W. P., Theory of linear antennas (Harvard University Press, 1956).

Simmons, G. J., A study of the error involved in the near use of a radio interferometer, Sandia Corporation Technical Memorandum No. 122-59(52) (unpublished). 